\title{
Vouchers for future kidney transplants to overcome "chronological incompatibility" between living donors and recipients
}

Veale, Jeffrey L ; Capron, Alexander M ; Nassiri, Nima ; Danovitch, Gabriel ; Gritsch, H Albin ; Waterman, Amy ; Del Pizzo, Joseph ; Hu, Jim C ; Pycia, Marek ; McGuire, Suzanne ; Charlton, Marian ; Kapur, Sandip ; Pycia,

Marek G

\begin{abstract}
Background: The waiting list for kidney transplantation is long. The creation of "vouchers" for future kidney transplants enables living donation to occur when optimal for the donor and transplantation to occur later, when and if needed by the recipient. Methods: The donation of a kidney at a time that is optimal for the donor generates a "voucher" that only a specified recipient may redeem later when needed. The voucher provides the recipient with priority in being matched with a living donor from the end of a future transplantation chain. Besides its use in persons of advancing age with a limited window for donation, vouchers remove a disincentive to kidney donation, namely, a reluctance to donate now lest one's family member should need a transplant in the future. Results: We describe the first three voucher cases, in which advancing age might otherwise have deprived the donors the opportunity to provide a kidney to a family member. These 3 voucher donations functioned in a nondirected fashion and triggered 25 transplants through kidney paired donation across the United States. Conclusions: The provision of a voucher to potential recipients whose need for a transplant makes them "chronologically incompatible" with their donors may increase the number of living donor transplants.
\end{abstract}

DOI: https://doi.org/10.1097/TP.0000000000001744

Posted at the Zurich Open Repository and Archive, University of Zurich

ZORA URL: https://doi.org/10.5167/uzh-147442

Journal Article

Published Version

Originally published at:

Veale, Jeffrey L; Capron, Alexander M; Nassiri, Nima; Danovitch, Gabriel; Gritsch, H Albin; Waterman, Amy; Del Pizzo, Joseph; Hu, Jim C; Pycia, Marek; McGuire, Suzanne; Charlton, Marian; Kapur, Sandip; Pycia, Marek G (2017). Vouchers for future kidney transplants to overcome "chronological incompatibility" between living donors and recipients. Transplantation, 101(9):2115-2119.

DOI: https://doi.org/10.1097/TP.0000000000001744 


\title{
Vouchers for Future Kidney Transplants to Overcome "Chronological Incompatibility" Between Living Donors and Recipients
}

\author{
Jeffrey L. Veale, MD, ${ }^{1}$ Alexander M. Capron, LLB, MA (Hon), ${ }^{2,3}$ Nima Nassiri, MD, ${ }^{3}$ Gabriel Danovitch, MD, ${ }^{1}$
} H. Albin Gritsch, MD, ${ }^{1}$ Amy Waterman, PhD, ${ }^{1}$ Joseph Del Pizzo, MD, ${ }^{4} \mathrm{Jim}$ C. Hu, MD, MPH, ${ }^{4}$ Marek Pycia, PhD, ${ }^{5}$ Suzanne McGuire, RN, BSN, ${ }^{1}$ Marian Charlton, $\mathrm{RN},{ }^{4}$ and Sandip Kapur, $\mathrm{MD}^{4}$

Background. The waiting list for kidney transplantation is long. The creation of "vouchers" for future kidney transplants enables living donation to occur when optimal for the donor and transplantation to occur later, when and if needed by the recipient. Methods. The donation of a kidney at a time that is optimal for the donor generates a "voucher" that only a specified recipient may redeem later when needed. The voucher provides the recipient with priority in being matched with a living donor from the end of a future transplantation chain. Besides its use in persons of advancing age with a limited window for donation, vouchers remove a disincentive to kidney donation, namely, a reluctance to donate now lest one's family member should need a transplant in the future. Results. We describe the first three voucher cases, in which advancing age might otherwise have deprived the donors the opportunity to provide a kidney to a family member. These 3 voucher donations functioned in a nondirected fashion and triggered 25 transplants through kidney paired donation across the United States. Conclusions. The provision of a voucher to potential recipients whose need for a transplant makes them "chronologically incompatible" with their donors may increase the number of living donor transplants.

(Transplantation 2017; 101: 2115-2119)

or transplantation to be a truly effective treatment for end-stage organ failure, the number of organ donors, and hence the supply of transplantable organs, must continue to grow. In June 2016, the White House Organ Summit identified several strategies for reaching this goal, including

\footnotetext{
Received 8 January 2017. Revision received 22 February 2017.

Accepted 12 March 2017.

${ }^{1}$ David Geffen School of Medicine at UCLA, Los Angeles, CA.

${ }^{2}$ Gould School of Law, USC, Los Angeles, CA.

${ }^{3}$ Keck School of Medicine, USC, Los Angeles, CA.

${ }^{4}$ New York-Presbyterian Weill Cornell Medical Center, New York, CA.

5 Department of Economics, UCLA, Los Angeles, CA.
}

Jeffrey L. Veale, MD, Suzanne McGuire, RN, BSN, Marian Charlton, RN, and Sandip Kapur, MD are on the National Kidney Registry Medical Board. None of the other authors disclose any funding or conflicts of interest.

J.L.V.: manuscript development, data interpretation, literature search, writing, figure development. A.M.C.: manuscript development, writing, literature search, medicolegal counsel. N.N.: manuscript development, literature search, writing, figure development, data interpretation. G.M.D.: manuscript development, writing. H.A.G.: manuscript development, writing. A.W.: manuscript development, writing. J.D-P.: manuscript development. J.C.H.: manuscript development. M.P.: manuscript development, writing, medical economics counsel. S.M.: manuscript development, writing. M.C.: manuscript development. S.K.: manuscript development.

Correspondence: Jeffrey L. Veale, MD, UCLA Kidney Transplantation Exchange Program, David Geffen School of Medicine at UCLA, 200 Medical Plaza, Suite 140, Los Angeles, CA 90095-1738. (JVeale@mednet.ucla.edu).

Copyright $\odot 2017$ Wolters Kluwer Health, Inc. All rights reserved.

ISSN: 0041-1337/17/10109-2115

DOI: $10.1097 / T P .0000000000001744$ removing financial and other disincentives for living donation and utilizing more nondirected living donors. ${ }^{1}$

Approximately $35 \%$ of patients with a relative or friend willing to donate a kidney are unable to accept that kidney due to biological incompatability. ${ }^{2,3}$ Kidney paired donation (KPD) was first used in the United States in 2000 as a means of allowing a transplant to occur in such circumstances. ${ }^{4}$ Based on identifying reciprocal compatibilities in two unmatched donor-recipient pairs, KPD occurs when a kidney from the donor in one pair is transplanted into the recipient in the other, and vice versa (Figure 1). When multiple swaps were combined into chains, the surgeries were initially carried out simultaneously but then moved to sequential transplants, with one donor giving to the recipient in another pair, whose donor then extends the chain by donating to the next recipient. ${ }^{5}$ With the introduction in 2007 of nondirected (altruistic) donors to initiate chains, KPD can extend over weeks to months and involve multiple centers in different cities (Figure 2). ${ }^{5}$

In 2008, a donor's need to give his kidney a week before the recipient in his incompatible pair was scheduled to receive a kidney from a nondirected donor occasioned the first departure from the usual KPD sequence. Without this alteration, the chain would not have existed, and seven downstream recipients would not have been transplanted. ${ }^{6}$ The utility of such out-of-sequence donations led the National Kidney Registry in 2012 to create the Advanced Donation Program, in which 21 transplant centers currently participate.

The voucher program is an expansion of KPD and is fundamentally different from the currently existing advanced 


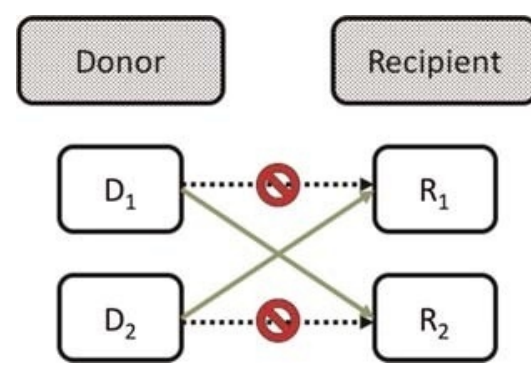

FIGURE 1. Kidney paired donation. A two-way swap is demonstrated, where the donor of the first incompatible pair donates to the recipient of the second incompatible pair and vice versa.

donation program in that voucher recipients are not in need of a kidney transplant and may never need a kidney transplant. Additionally, donors in the voucher program function as nondirected donors by initiating chains without adding a paired recipient to the current chain. When a voucher is redeemed, a future chain of transplantation will end by providing the voucher recipient with a compatible kidney.

\section{A Solution for Chronological Incompatibility}

Besides biological incompatibility, a willing donor may also find that "chronological incompatibility" stands in the way of providing a kidney to a relative or friend because the optimal time to donate a kidney occurs long before the intended recipient needs a transplant. In this proof-of-concept report, we describe the first three cases utilizing a "voucher" to overcome chronological incompatibility and, in the process, to trigger KPD chains. These 3 donors enabled 25 kidney transplantations to occur across the United States. Vouchers are recorded in the National Kidney Registry's database and represent a commitment to provide the donors' intended recipients (who do not currently need a transplant) priority to receive a kidney from a living donor at the end of a future chain (Figure 3).

\section{Case 1}

A 4-year-old child with chronic kidney disease (CKD) attributed to a poorly functioning solitary native kidney was expected to require a renal transplant in 10 to 15 years. The child's grandfather, a 64-year-old judge, was prepared to donate a kidney when his grandson needed it. Rather than allow his candidacy as a living donor to disappear as he aged, the grandfather approached the UCLA Kidney Transplant Program to discuss the possibility of donating a kidney immediately, with the pledge that when needed his grandson would receive priority in getting a living donor transplant.

The UCLA Living Donor Committee and the National Kidney Registry Medical Board approved the voucher that embodies this pledge, with several stipulations: it has no monetary value and can be used only by the grandson and not transferred to another patient. Further, all parties involved agreed that while the Registry is committed to taking the steps necessary to provide a transplant, the voucher cannot ensure that a suitable kidney would be available. These points were explicitly stated in the informed consent documents, which were discussed with and signed by both the donor and the guardian of the intended recipient. A thorough evaluation of the donor, including psychological testing, established that he was medically suitable, highly educated, clearly informed, and that he understood that his grandson would be prioritized for, but not guaranteed, a future living donor kidney.

In December 2014, the grandfather underwent a living donor nephrectomy at UCLA, initiating a transplant chain with three recipients, who discontinued dialysis and were removed from the deceased donor waitlist. The grandson's renal status has not yet warranted redemption of the voucher.

\section{Case 2}

In 2007, when she was 10, a girl underwent a kidney transplant from a living donor. Nine years later, her graft function remained excellent, but her 52-year-old father wanted to donate a "back-up" kidney in case his daughter's allograft eventually failed. The father welcomed the use of the voucher because he wanted to make his donation before he became too old to do so. In August 2015, at New York-Presbyterian Weill Cornell Medical Center, the father donated a kidney, triggering a chain of eight transplants and assuring that if his
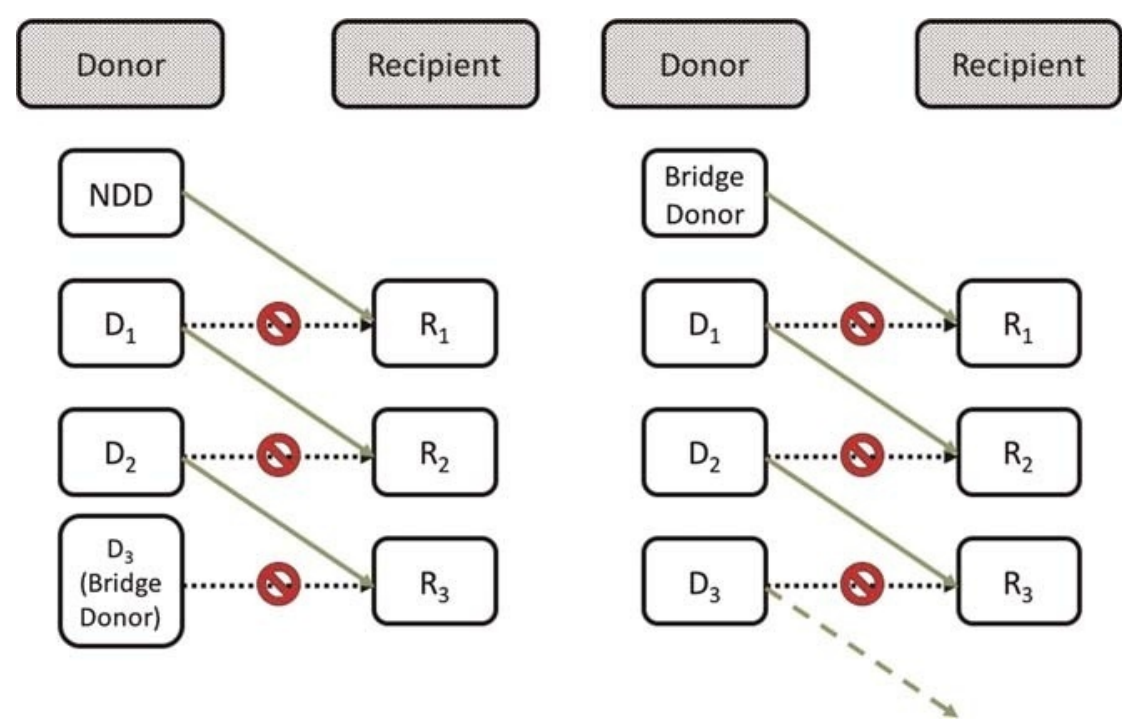

FIGURE 2. Chains of transplantation. A nondirected donor donates to a recipient of an incompatible pair. Rather than the chain terminating in the donation to a recipient on the deceased donor waitlist, the last donor serves as a bridge, propagating the chain. 


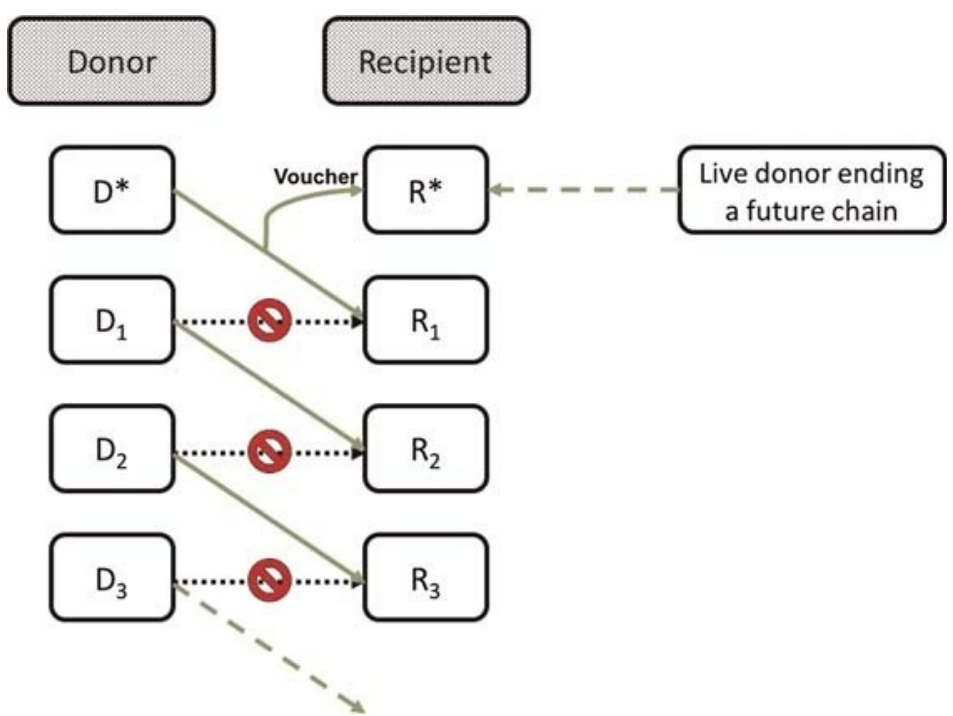

FIGURE 3. Voucher program. A currently viable live donor ( $\left.D^{*}\right)$ initiates a chain of transplantations like a nondirected donor in exchange for a voucher provided to a recipient $\left(R^{*}\right)$ who may need a kidney in the future. The transplantation chain continues per current standards. Arrows reflect the intended (dashed, black arrow) or actual (solid, green arrow) flow of kidneys within a chain of transplantation. A future chain of transplantation (dashed, green arrow) will end in $\mathrm{R}^{\star}$, when the voucher is redeemed.

daughter needs a second allograft in the future, she will be prioritized for a living donor from the end of a future KPD chain.

\section{Case 3}

Due to the young age of the patient in the previous case, her clinicians believe that she may someday require a third kidney transplant to avoid having to go onto dialysis. Therefore, in May 2016, the patient's 60-year-old aunt also donated a kidney at New York-Presbyterian Weill Cornell Medical Center, initiating a chain of 14 kidney transplants while also providing a second voucher for her niece.

\section{DISCUSSION}

\section{Evolution of Vouchers}

The growth of KPD in the United States has been driven by innovations that aim to remove logistical and bureaucratic barriers, especially those that disincentive donation. Advanced donation emerged as a short-term accommodation for donors with a deadline to return to work or the like, which leads to the donation sequence being altered prospectively. ${ }^{6}$ In contrast, when an unexpected recipient event delays transplantation, the donor-recipient pair is often placed into the advanced donation program retrospectively. ${ }^{8}$ In either case, at the time of their donor's nephrectomy, paired recipients in the advanced donation program need a transplant, which typically occurs within several weeks to months of the donation. ${ }^{6,8}$

Vouchers represent another expansion of KPD and exhibit two fundamental differences from standard advanced donations. First, the intended recipients are not yet in need of a kidney transplant and may never need a kidney transplant. Second, voucher donors function as nondirected donors who can initiate chains because they do not add a paired recipient to the current chain. When voucher holders do not progress to end-stage renal failure, die first from other causes, or become medically unsuitable for transplantation, their vouchers would not be redeemed, so the donor would remain nondirected. Moreover, as the third case demonstrates, the opportunity to obtain a voucher can stimulate multiple donations for a patient who may need one or more transplants in a lifetime, increasing the likelihood that some of these vouchers will never be redeemed. When and if a voucher is redeemed, the donation becomes equivalent to any other KPD donor's, albeit separated from the recipient's transplant by an extended period.

In what follows, we explain how vouchers could substantially increase the number of KDP chains and thus remove patients from the deceased donor waitlist. When adopting vouchers, however, networks of transplant centers will need to consider the effects on nondirected donation and guard against the potential for organ vending, donors gaming the system, and unsustainability.

\section{Vouchers' Potential}

Vouchers can motivate many groups of people to donate. As illustrated by the three cases, one group consists of donors who fear that with advancing age they will become unable to donate to a specific person, who does not yet need a first or replacement transplant. Similarly, people who are anticipating life events such as relocation, marriage, or childbirth that could affect their donation candidacy can donate a kidney now to do good in the future, when that help is needed. If only a fraction of the 40 million CKD patients in the United States had a donor like the grandfather in case 1, then tens of thousands of high-quality organs would enter the system. Cases 2 and 3 suggest that another great potential for vouchers resides with the family and friends of the 200000 transplant recipients in the United States whose current allograft may eventually fail. ${ }^{9}$ Many of these people are well informed about transplantation and may indeed have been evaluated and found suitable to donate when the patient received his or her current allograft. Were even a small percentage to donate, the supply of living donor organs could be greatly increased, especially because, as cases 2 and 3 illustrate, a single patient may inspire multiple acts of donation, decreasing the probability for voucher redemption. 
Another group of donors would be produced by vouchers' ability to overcome a major disincentive to nondirected donation, namely, reluctance to donate lest one's currently healthy spouse or child needs a transplant in the future. Although the voucher program will create the burden of having to provide kidneys for recipients designated by some number of people who would have become nondirected donors anyway, it will also motivate others whose altruistic tendencies are held back by their concern that they need to hold on to their ability to donate. If several of these potential nondirected donors are reassured by vouchers, the number of patients who receive transplants will increase not only because many vouchers will never be redeemed (because relatively few healthy people develop kidney failure) but more importantly because each nondirected donor enables an average of 4.7 recipients with incompatible donors to receive a transplant from a living donor. ${ }^{10}$

\section{What Vouchers Are and Are Not}

Vouchers are a means of overcoming chronological incompatibility between donor and recipient pairs, and removing a disincentive for donation. They are supplements to, not substitutes for, the normal process of screening donors medically and psychosocially, as well as informing them about what is involved in donation. Informed consent for both donor and recipient are of utmost importance to the success of the program. Besides disclosing the potential risks and burdens associated with organ donation, transplant teams need to ensure that donors and intended recipients understand that while vouchers provide priority for a living donor transplant should that need materialize, they cannot guarantee that one will be available. Vouchers also neither help nor hurt recipients' status on the deceased donation waitlist. The matching algorithm of the National Kidney Registry is focused on the creation of chains with clusters that maximize the number of transplants facilitated. A candidate redeeming a voucher is the third of six categories in the current priority, following, first, a nondirected donor who needs a transplant, having previously donated as part of the National Kidney Registry, and, second, mending a chain where a recipient did not receive a kidney because of a real-time swap failure. ${ }^{11}$ When the voucher is redeemed, a future chain will end with the identified voucher recipient.

During the evaluation process, two other points need to be clear. First, the original donor must affirm that he or she has received no money or "valuable consideration" for donating the organ that will generate the voucher. Second, the voucher has no cash value and cannot be sold or bartered to another person. When the person listed on an unredeemed voucher dies or becomes permanently ineligible for kidney transplantation, the voucher expires. To avoid surreptitious paid exchanges, the identity of voucher recipients, who are selected prior to donor nephrectomy, must be recorded (photo identification, $\mathrm{ABO}$ blood group, and tissue type) to permit confirmation when vouchers are redeemed.

In the three reported cases, only virtual vouchers-that is, electronic records that can be called up from the network's database when needed-were created. A physical voucher could provide recipients with secure evidence that the pledge of help in getting a renal allograft will not be withdrawn. Yet a printed document might generate misunderstanding because it might seem more like other sorts of vouchers, such as gift vouchers with a specified monetary value or certificates that entitle the bearer to a good or service, based on having purchased something else. In any case, the term itself is appropriate because it has traditionally meant any document that provides proof of a fact. Here, the voucher attests to the original donation having generated a credit with a transplant network so that, should the medical need arise in the future, a specified person will have priority for a spot at the end of a KDP chain.

\section{Further Points to Consider}

Another concern is that some voucher participants may try to "game the system" by donating before their intended recipient needs a transplant to give the recipient a chance to receive a better organ than anticipated through traditional KPD. Like any potential recipient, a candidate redeeming a voucher may refuse an offered kidney in hopes of getting a better one, but doing so comes with the burden of more time on the waitlist. Even without such manipulation, in situations like case 1 , the potential recipient is likely to receive a considerably better kidney (if needed) in a few years than the kidney donated by his 64 -year-old grandfather. However, from the viewpoint of the whole system, the kidney donated by the grandfather was a very good one; it added significant net value by unlocking two younger, previously unutilized living donors from their paired incompatibilities, thereby removing three patients from the constraints of dialysis.

Any organization responsible for a voucher program would be concerned if requests exceed the availability of chain-end donors, resulting in an inability of potential recipients to redeem their vouchers. The likelihood of this occurring should remain low as each voucher donor will start a chain in which other donors, freed from their cross-match incompatibilities, create additional chain-ending donors. ${ }^{12,13}$ Additionally, only a portion of vouchers will be redeemed due to the designated recipient's death or lack of progression to severe kidney disease. Voucher programs will also need a policy to choose among several potential recipients who all match the same chain-ending donor. Priority could go to the patient with the highest panel of reactive antibody level or the one who has held a voucher for the longest time. Further, as with any deceased or living donor program, highly sensitized and "O" blood group candidates will be a concern for voucher programs. The more widely the program is adopted, the more likely that suitable donors will be found for all recipients redeeming vouchers.

A final issue is how to ensure appropriate follow-through, given the voluntary nature of the networks through which KPD is currently managed and the extended timeframe for voucher redemptions. The National Kidney Registry seems to be an appropriate organization to satisfy these voucher contracts, as it is the leading multicenter KPD organization, composed of over 70 centers, and has facilitated over 2000 transplants. Transplant centers, as protectors of the interests of their patients, should ensure that, were the Registry to undergo a change of control, any successor organization will have the same (or better) capacity to honor all the obligations created by any outstanding vouchers. Given the role of the United Network for Organ Sharing (UNOS) as the official Organ Procurement and Transplantation Network, it would be reasonable for it to establish rules to provide voucher recipients with deceased donors if they cannot be placed at the end 
of a living donor chain, especially considering that the $\mathrm{Na}$ tional Kidney Registry has contributed hundreds of "chainending" living donor kidneys to recipients on the deceased donor waitlist. Obtaining a variance from UNOS is likely to take several years, during which the voucher program should move ahead under its existing auspices as an innovative- - and sustainable-means of increasing transplants.

Iterations of the many potential situations that may arise beyond the current framework must be addressed as encountered, with appropriate regulatory changes made as needed. This has been the experience with each evolutionary step in KPD to date, from the establishment of swaps, chains of transplantation, out-of-sequence chains, and now, with vouchers.

\section{CONCLUSION}

The creation of vouchers for future kidney transplants is a substantial improvement in KPD that enables living donation to occur when optimal for the donor and transplantation to occur later, when and if needed by the recipient. The original donation functions in a nondirected fashion, triggering a KPD chain, such as those that followed the 3 proof-of-concept cases presented here, which together resulted in 25 transplants nationally. If any of the vouchers in these cases are redeemed, a future chain will end with the identified voucherrecipient, thus overcoming the "chronological incompatibility" between the patient and his or her donor. If a voucher is never redeemed, then the donor will have truly been a nondirected donor, with all that implies for increasing the number of transplants. This innovation could, if broadly adopted, significantly increase the number of living donor transplants performed and thereby reduce the waiting time for a deceased donor transplant.

\section{ACKNOWLEDGMENTS}

The authors thank Rhiannon Kure for initial interviews with clinicians, and Amanda Chi for assistance with early rendering of figures.

\section{REFERENCES}

1. Pruett TL, Chandraker A. The White House Organ Summit: what it means for our field. Am J Transplant. 2016;16:2245-2246.

2. Gentry SE, Montgomery RA, Swihart BJ, et al. The roles of dominos and nonsimultaneous chains in kidney paired donation. Am J Transplant. 2009;9:1330-1336.

3. Segev DL, Gentry SE, Warren DS, et al. Kidney paired donation and optimizing the use of live donor organs. JAMA. 2005;293:1883-1890.

4. Ellison B. A Systematic review of kidney paired donation: applying lessons from historic and contemporary case studies to improve the US model. Wharton Research Scholars. 2014;107.

5. Rees MA, Kopke JE, Pelletier RP, et al. A nonsimultaneous, extended, altruistic-donor chain. N Engl J Med. 2009;360:1096-1101.

6. Butt FK, Gritsch HA, Schulam P, et al. Asynchronous, out-of-sequence, transcontinental chain kidney transplantation: a novel concept. Am J Transplant. 2009;9:2180-2185.

7. National Kidney Registry. Advanced Donation Program (ADP) Participating Centers. https://www. kidneyregistry.org/referral/adp-centers. Updated 2017.

8. Flechner SM, Leeser D, Pelletier R, et al. The incorporation of an advanced donation program into kidney paired exchange: initial experience of the National Kidney Registry. Am J Transplant. 2015;15:2712-2717.

9. Wolfe RA, Ashby VB, Milford EL, et al. Comparison of mortality in all patients on dialysis, patients on dialysis awaiting transplantation, and recipients of a first cadaveric transplant. N Engl J Med. 1999;341:1725-1730.

10. Melcher ML, Leeser DB, Gritsch DB, et al. Chain transplantation: initial experience of a large multicenter program. Am J Transplant. 2012;12: 2429-2436.

11. National Kidney Registry. Medical Board Policies. http://www. kidneyregistry.org/transplant_center.php\#policies. Published 2016.

12. Melcher ML, Veale JL, Javaid B, et al. Kidney transplant chains amplify benefit of nondirected donors. JAMA Surg. 2013;148:165.

13. Danovitch G, Veale J, Hippen B. Living donor kidney donation in the United States: quo vadis? Am J Transplant. 2010;10:1345-1346. 